JUAN LUIS CONDE

(Universidad Complutense de Madrid)

DAVID SEVILLANO LÓPEZ

(Universidad Complutense de Madrid)

ZIQIAO MENG

(Universidad Carlos III de Madrid)

\title{
LA TÉCNICA YIN: TEORÍA Y PRÁCTICA DEL DISCURSO PERSUASIVO EN CHINA DURANTE LOS REINOS COMBATIENTES (SS. V-III A.C.)
}

\section{The Yin Technique: Theory and Practice of Persuasive Discourse in China during the Warring States Period (5th to 3rd Centuries BC)}

\begin{abstract}
The authors of this paper translate from Classical Chinese and comment on various texts that are intended to illustrate the relationship between the theory and practice of persuasive discourse during the Warring States period (5th to 3 rd centuries BC). The texts come from two different works: Zhanguoce, a compilation of pre-Qin documents made by Liu Xiang in the Han period (late 1st century BC), and Lüshi Chunqiu, an encyclopedic collection undertaken under the instructions of Lü Buwei, Qin State Minister, in the 3rd century BC. The political suasoria (shui has the counselor and the king as protagonists par excellence. In the anecdotes that provide concrete examples of the rhetorical exercitatio, an approach decidedly marked by the unequal power relations between both of them is observed, where dialogue dominates on monologue. Under such conditions, the persuader develops an indirect strategy that, disdaining any virtuosity of elocution, tries first and foremost not to challenge the expectations of his one-person audience.
\end{abstract}

KEY WORDS: Ancient Chinese Rhetoric, persuasive discourse, Zhanguoce, Lüshi Chunqiu, translation.

RESUMEN: Los autores del artículo traducen del chino clásico y comentan varios textos que pretenden ilustrar la relación entre la teoría y la práctica del discurso persuasivo durante el período de los Reinos o Estados Combatientes (ss. V-III a. C.). Los textos proceden de dos obras diferentes: Zhanguoce, una compilación de documentos pre-Qin realizada por Liu Xiang en época Han (finales del siglo I a.C.), y Lüshi Chunqiu, una colección enciclopédica emprendida bajo las instrucciones de Lü Buwei, ministro del Estado de Qin, en el siglo III a.C. La suasoria política (shui) tiene como protagonistas por excelencia al consejero y al soberano. En las anécdotas que proporcionan ejemplos concretos de la exercitatio retórica se observa un planteamiento decididamente marcado por las desiguales relaciones de poder entre ambos, donde domina el diálogo sobre el monólogo. En esas condiciones, el suasor despliega una estrategia indirecta que, desdeñando el virtuosismo elocutivo, pretende en todo momento no violentar las expectativas de su auditorio unipersonal.

Palabras Clave: Retórica antigua china, discurso persuasivo, Zhanguoce, Lüshi Chunqiu, traducción.

Fecha de Recepción: 7 de julio de 2020.

Fecha de Aceptación: 1 de octubre de 2020. 


\section{TALIA DIXIT 15 (2020), 101-121}

\section{La suasoria politica durante los Reinos Combatientes desde un punto de vista comparativo}

Durante los últimos años hemos venido publicando diversos artículos con el objetivo de presentar las características más destacadas de la retórica china antigua posibilitando al mismo tiempo, gracias a traducciones de textos inéditos en castellano, un debate más extenso en nuestra lengua. Lo cierto es que esas traducciones y su comentario afectaban a aspectos que podríamos describir con el viejo concepto romano de ars, es decir, planteamientos teóricos rastreados por diversas obras pertenecientes a un mismo período histórico: el conocido como Reinos (o Estados) Combatientes (convencionalmente 475-221 a. C.).

Nuestra pretensión en este artículo es proporcionar a los estudiosos de la retórica en general y a quienes puedan estar interesados en un conocimiento más detallado del fenómeno en la China clásica, pero no necesariamente sinólogos, un lado de la cuestión que hasta ahora no habíamos ilustrado: lo que, recurriendo también a los términos de la retórica latina, habría que identificar como exercitatio, es decir, la práctica activa de la persuasión. Para ello hemos reunido en este artículo traducciones de dos fuentes diferentes con materiales de esa misma época y cuyas características se exponen de forma sumaria para contextualizar adecuadamente los textos que comentamos.

Este período coincide con el final de lo que habitualmente se identifica con el clasicismo chino, que estaría así demarcado por este período y el inmediatamente anterior, Primavera y otoño (s. VIII-V a. C.). El nombre de Reinos Combatientes [战 国 pinyin: Zhàn guó] resulta apropiado para una época convulsa y singularmente novelesca en el que siete reinos, liberados de la tutela que la dinastía Zhou oriental había ejercido previamente, luchan entre sí por imponer su hegemonía o por conservar una precaria independencia. A ese panorama de conflictos armados externos se añadía una vida política interna marcada por las luchas de poder y las intrigas, a menudo con consecuencias cruentas. Se explica así la sensación de caos que trasmiten de manera recurrente las obras de los escritores del período y su añoranza de un orden estable. La etapa histórica desembocará en la unificación que en el año 221 a. C. impone el llamado Primer Emperador (Qin Shihuang). Ese fue también el final de un mundo de efervescencia intelectual y que, por sus propias características, representa una época vibrante desde el punto de vista de la retórica política, entendida aquí como teoría y práctica del uso persuasivo del lenguaje y sus estrategias en el marco de las relaciones de poder.

Para entender en sus dimensiones esenciales este particular discurso persuasivo durante el período habría que tener en cuenta al menos tres aspectos: 


\section{TALIA DIXIT 15 (2020), 101-121}

1. Las condiciones de la ejecución oratoria difieren de las europeas.

En la China de los Reinos Combatientes la forma más característica de la suasoria política $(\text { shui 说 })^{1}$ se desarrolla en una situación muy diferente de la oratoria pública ante un auditorio de iguales o subalternos, como es característico en las instituciones adoptadas por la democracia ateniense en el siglo V a. C. o la República de Roma hasta el I a. C.: el practicante chino por excelencia de lo que la teoría retórica europea denomina discurso deliberativo o político es el consejero que intenta convencer a un auditorio compuesto por un único oyente, el soberano. Esta relación retórica se produce siempre en privado, en el interior del palacio real. La superioridad del rey sobre su consejero está plasmada escénicamente: el monarca le observa desde arriba, aposentado en su trono, que está en una posición elevada e inexcusablemente orientado hacia el sur. Como polo débil de la relación de poder, el consejero, situado frente al soberano, mantiene una postura humillada con los ojos vueltos al norte $y$, al hablar, no sólo se juega la capacidad de persuasión como el orador griego o el romano, sino también, literalmente, la vida. ${ }^{2}$

2. El discurso persuasivo se concibe más como diálogo que como monólogo.

Este contexto impone una drástica contraposición con los presupuestos de la tratadística retórica clásica de raíz greco-latina: la doctrina teórica que se dispensa no enseña tanto a persuadir auditorios amplios que guardan silencio mientras se pronuncia un monólogo ante ellos como interlocutores individuales que participan en un intercambio. No se ocupa tanto de la alocución, como de la interlocución. No instruye, pues, en el monólogo, sino en el diálogo. Puede entenderse, en suma, como una dialéctica en el sentido socrático: un estudio de la conversación. Y, sin embargo, aunque no se trate de un monólogo mantenemos la expresión "discurso persuasivo" porque el suasor debe organizar una estrategia para ese intercambio dialogado: es a ese planteamiento estratégico subyacente, destinado a dirigir la conversación, al que cabe denominar "discurso".

En esa diferencia de orden socio-espacial (un escenario privado frente a uno público, un diálogo frente a un monólogo) es donde el sinólogo francés François Jullien encuentra el origen del divergente desarrollo teórico de la retórica en Europa y China: en razón de sus respectivas circunstancias, el minucioso análisis de las técnicas del discurso que conoció el mundo grecolatino habría sido sustituido en China por un interés fundamental en los aspectos psicológicos de la relación discursiva. ${ }^{3}$ En esas condiciones, el suasor es considerado como un oyente

1 Este carácter representa también al verbo shuo, que significa "hablar" o "decir", con una evidente conexión etimológica.

${ }^{2}$ Cf. Conde y Zhao (2016: 32).

3 Jullien (1999: 246-7). 


\section{TALIA DIXIT 15 (2020), 101-121}

especializado y la tratadística subraya su capacidad de escucha y observación como parte esencial de su eficacia retórica.

3. El uso persuasivo del lenguaje y su teoría han sido históricamente sospechosos para la ortodoxia confuciana.

Jullien ha hablado, como consecuencia de sus particulares circunstancias, de "anti-retórica" para una teoría china cuyo objetivo, en lugar de la persuasión (que sería propia de la retórica europea), sería la manipulación. Esa visión negativa ha recibido numerosas críticas y objeciones, pero podría decirse que ha contado con opiniones parecidas incluso en el ámbito chino. Confucio y su escuela manifestaron en numerosas ocasiones una desconfianza hacia el poder de la palabra y su capacidad de engaño. Quizá porque desde muy temprano esa corriente de pensamiento se ha identificado con lo propiamente chino, ha podido afirmarse que la retórica no es un arte desarrollado en aquel mundo cultural. Pero habría que decir que, más que antichina, la retórica surgió anti-confuciana o, al menos, a-confuciana: enseñaba cosas que ponían en entredicho o entraban en conflicto abierto con una moral exigente que promovía la sinceridad y la supremacía de los principios éticos al tiempo que condenaba la búsqueda de ventaja o eficacia a cualquier precio. Por explicarlo en los términos de una vieja polémica latina: al insistir sobre el vir bonus, el confucianismo ponía implícitamente bajo sospecha al peritus dicendi. ${ }^{4}$

Hay que tener en cuenta que la formación retórica (y, por tanto, sus manuales) estaba destinada fundamentalmente a quienes pretendían hacer carrera como consejeros y diplomáticos, cuya tarea fundamental consistía en convencer al rey de que tomase determinadas decisiones. Al plantearse desde esa perspectiva, se temía que la teoría pudiera servir para que, en un sentido más amplio, los subalternos sacasen partido de los jefes o superiores y ésa es una de las razones por las que los libros que la enseñaban han sido históricamente considerados peligrosos. ${ }^{5}$ Este conflicto estuvo siempre ahí, pero se agudizaría a partir de la época Han (s. II a. C.), cuando el confucianismo se convertiría en la doctrina oficial del poder imperial chino y piezas fundamentales de la tratadística retórica fueron excluidas del canon de lecturas y de la ortodoxia educativa. Ése sería el caso, específicamente, del Guiguzi

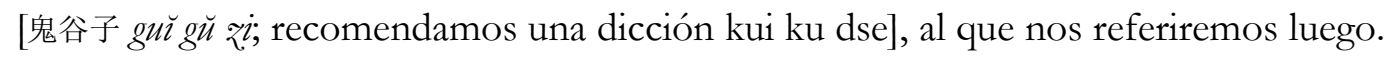

\section{El Guiguzi puesto en práctica en el Zhanguoce: Chu Long aconseja a la reina regente de Zhao}

Parece haberse asentado en castellano el título Anécdotas de los Reinos Combatientes para la obra que se conoce en chino bajo el nombre de tres caracteres

\footnotetext{
${ }^{4}$ Véase Li (2017: 27).

${ }^{5}$ Véase Wu y Swearingen (2016: 18).
} 
zhàn guó cè 战国 策 [dicción propuesta: chan kuo tse; en adelante ZGC]. La traducción resulta irreprochable desde el punto de vista histórico: con excepción de un solo incidente, los acontecimientos de los que se ocupa el libro habrían tenido lugar entre el año 454 y el 221 a. C., ${ }^{6}$ lapso temporal que coincide prácticamente con el período de los Reinos Combatientes. Compuesto por una recopilación de materiales procedentes de diversas fuentes documentales, su contenido reúne un extenso catálogo de episodios bélicos y ejemplos de diplomacia y consejística procedente de los siete Estados en que se dividía China durante ese período histórico.

Sin embargo, la interpretación del título original sigue sometida a debate por lo que respecta a la palabra cè 策: algunos estudiosos creen que debería interpretarse en referencia a las tablillas de bambú o madera que se usaban en la escritura y, por tanto, significaría "documentos". Sin embargo, Liu Xiang (79-8 a. C.), quien fue el primer compilador y puso título a la obra, señala expresamente que el libro contiene materiales empleados por los diplomáticos de los Reinos Combatientes para aconsejar a esos Estados que los contrataban urdiendo tramas e intrigas ${ }^{7}$ en su provecho. Habría, por lo tanto, buenas razones para entender el título con un significado cualquiera del amplio espectro de sinónimos que tiene el castellano para expresar la idea: añagazas, ardides, argucias, artimañas, tretas o estratagemas.

Cualquiera de esos términos cargados connotativamente daría cuenta del objetivo de obtener ventaja de una manera que, como sucede también con las enseñanzas del Guiguri, ha sido considerada en contradicción con los principios de la moral confuciana. ${ }^{8}$ Como consecuencia, los estudiosos confucianos, incluyendo al propio Liu Xiang, han condenado sus implicaciones morales y políticas. El compilador del ZGC denuncia como una práctica habitual de la época el uso de "medios indignos con el único propósito de conseguir el poder". ${ }^{9}$ Como él, otros estudiosos posteriores, mientras desaprobaban las lecciones morales del libro, se sintieron sin embargo seducidos por la sutileza retórica y su ágil estilo literario. "Veneno en una comida deliciosa": la opinión que expresa Lu Longqi, un intelectual de la época Ming (s. XVII), subsume con elegancia la actitud tradicional hacia la obra. ${ }^{10}$

Igual que frente al aspecto moral, el aprecio por el logro literario de la obra se hace destacar habitualmente también frente a su valor histórico. Ése es el parecer de Tsuen-hsuin Tsien, autor del apartado correspondiente en la obra colectiva Early Chinese Texts, quien escribe:

\footnotetext{
${ }^{6}$ Tsien (1993: 4).

7 "Plans and plots", Tsien (1993: 1-2).

${ }^{8} \mathrm{Al}$ respecto, véase Conde y Zhao (2018).

9 Tsien (1993: 1)

${ }^{10}$ Cf. Tsien (1993: 1).
} 


\section{TALIA DIXIT 15 (2020), 101-121}

The unique style and content of the book set a precedent in explaining the strategies of power politics by means of diplomatic argument. The clever methods of persuasion with their vivid characterizations, their wit and humour impart to the work a literary merit that is higher than its historical value. ${ }^{11}$

Dado que entre la fecha de los acontecimientos más recientes que se mencionan en el ZGC y la compilación de Liu Xiang, a finales del siglo I a. C., trascurren dos siglos, es posible que se haya incluido en ella algún material escrito con posterioridad al período de los Reinos Combatientes. Sin embargo, el grueso de los textos de la compilación forma parte de los escasos y afortunados documentos pre-Qin, que sobrevivieron al fanatismo destructivo del Primer Emperador. Nuestra referencia es la edición crítica comentada de Fan Xianyong, ordenada por Estados y publicada originalmente en 2011, en su reedición de 2018. El pasaje original que hemos traducido se localiza en la cuarta parte del reino de Zhao, en el capítulo [piàn] 21, texto número 18, entre las páginas 1230 y 1240 del libro de Fan.

Una de las fuentes principales del material que compiló Liu Xiang eran documentos procedentes de los archivos de los Estados que, aunque no eran historias oficiales, remiten a hechos constatados o confirmados en otros documentos de carácter histórico. En concreto, la anécdota del ZGC que hemos traducido al castellano puede datarse gracias a las referencias que proporciona el historiador Sima Qian. En su edición comentada, Fan Xianyong remite a las Memorias Históricas [Shïji] de este autor de época Han para situar el acontecimiento en el año 265 a. C., tras fallecer el rey Huiwen del Estado de Zhao. Heredó entonces la corona su hijo Xiaocheng, aún menor de edad. Viéndose en la necesidad de ayudar a su hijo, la viuda del difunto rey, conocida como Wei, inició una carrera política como regente del reino en su condición de tài hòu o reina madre:

Poco después de hacerse cargo de los asuntos públicos la reina regente de Zhao, el reino de Qin lanzó un virulento ataque contra su territorio. Entonces Zhao pidió ayuda a Qi. La respuesta de Qi fue: "Enviaremos un ejército a condición de que entreguéis como rehén al Príncipe de Chang'an.”12 La regente no accedió. Los consejeros ${ }^{13}$ insistían en que cambiase de opinión, pero la regente les advirtió:

- iA quien vuelva a proponerme que entregue al príncipe como rehén le escupiré en la cara!

11 Tsien (1993: 1).

12 Cháng àn jün: título nobiliario del menor de los cuatro hijos de la reina. Traducimos el tratamiento jün como "Príncipe" en atención a este contexto particular.

13 Dà chén: literalmente "alto servidor". Describe en general a los funcionarios de rango superior. 
Su primer ministro, ${ }^{14}$ el general Chu Long, pidió una audiencia con la regente, quien le esperaba muy irritada. Cuando el general entró en la sala recorrió muy despacio el trayecto protocolario ${ }^{15}$ hasta situarse frente a ella.

-A vuestro servidor la edad no le permite caminar bien: por eso he tardado tanto en llegar hasta aquí —explicó para disculparse-. Pensando en mi propia dolencia, me preocupaba que la salud de Vuestra Majestad pudiera estar afectada también por la debilidad, y por eso deseaba venir a veros.

La regente replicó:

- Para moverme yo cojo siempre mi palanquín.

Chu Long le preguntó:

— ¿Vuestra Majestad tiene buen apetito normalmente?

La regente le contestó:

- Me alimento con gachas de arroz.

El general dijo entonces:

-Vuestro servidor ha perdido el apetito hace tiempo, por eso se esfuerza por caminar cada día tres o cuatro $l^{16}$ para mejorar en lo posible las ganas de comer.

La reina regente repuso:

—A mí no me resulta posible — su furia había amainado un poco-.

El primer ministro empezó a contar:

-El menor de mis hijos, Shu Qi, no ha heredado las virtudes de nuestros antepasados, pero en mi fuero interno le tengo mucho cariño. Me encantaría que pudiera incorporarse a la guardia real y defender así el palacio. Me atrevo humildemente a solicitarlo.

La regente contestó:

—Lo acepto con mucho gusto. Y, ¿cuántos años tiene ya?

Chu Long respondió:

-Sólo quince. Es demasiado joven, pero desearía confiároslo antes de que me llegue la hora.

La regente repuso: pequeño...

—Ah, también usted, siendo hombre, siente debilidad por su hijo

A lo que él respondió:

- Más que una mujer.

La regente se rió:

14 Zuǒ shì: literalmente "el General de la Izquierda". La posición de shì, de origen militar, se complementaba con un yòu shi, "General de la derecha", y sus funciones abarcaban una amplia gama de contenidos: actividades ceremoniales, asuntos militares, relaciones exteriores, administración del estado, etc. Por comodidad para nuestra traducción desdoblamos el origen y sus funciones.

${ }^{15} \mathrm{La}$ aproximación del alto funcionario al trono de la reina debía atenerse a un movimiento protocolario caracterizado por pasitos cortos y rápidos.

${ }^{16}$ La extensión de la medida de distancia li ha variado a lo largo del tiempo. Bajo la dinastía Han, en tiempos del compilador Lu Xiang, 1 li equivalía a 415,8 metros. 
—iMás que una madre es imposible! El amor de una mujer por su hijo menor es incomparable.

Chu Long dijo:

-Pero sospecho que Vuestra Majestad quiere más a su hija, la reina de Yan, que a su hijo pequeño, el Príncipe de Chang'an.

—QQué va! —replicó ella—. Está usted equivocado: lo que siento por mi hijo no se puede superar.

El general argumentó:

-Cuando los padres quieren a sus hijos hacen planes para su futuro. Cuando Vuestra Majestad se despidió de su hija prometida con el rey de Yan, le ayudó a subir al carro llorando, entristecida aún más por la idea de que ella se iba tan lejos. Pero una vez que se marchó, nunca dejó Vuestra Majestad de preocuparse por ella ni de rezar en las ceremonias pidiendo: "Que nunca vuelva si eso implica que ha sido destronada". ¿No significa eso que Vuestra Majestad está planeando que algún día los descendientes de su hija hereden el trono?

-Así es — respondió la reina-.

—Ahora bien —preguntó Chu Long-, si nos remontamos tres generaciones hasta los orígenes del Estado de Zhao, de cuantos hijos de la familia real recibieron un título nobiliario, chay alguno cuyos descendientes conserven la herencia? ${ }^{\text {17 }}$

Ella contestó:

-Ni uno.

Chu Long insistió:

—No sólo en Zhao: ¿hay alguno en cualquier otro de los Estados?

—A mí no me consta — respondió ella—.

- Hemos visto — repuso Chu Long — que a veces la adversidad les golpeó personalmente a ellos y a veces se ensañó con sus descendientes. ¿Debemos concluir entonces que los herederos de los poderosos siempre son unos ineptos? Lo que pasa es que tienen una posición de privilegio sin haber logrado nada por sí mismos, un gran estipendio sin haberlo merecido y un enorme patrimonio. Vuestra Majestad ha otorgado a su hijo el título de Príncipe de Chang'an, le ha concedido fértiles terrenos como feudo y le ha regalado un gran patrimonio, pero no le ha dado oportunidad para hacer méritos en provecho del Estado: el día en que Vuestra Majestad desaparezca, ¿con qué apoyo podrá contar el Príncipe de Chang'an en Zhao? Yo creía que Vuestra Majestad no había hecho planes a largo plazo para el Príncipe de Chang'an, y por eso pensaba que su amor hacia el príncipe no era igual que hacia su hija, la reina de Yan.

La reina regente concedió: príncipe.

-De acuerdo. Provea usted de la manera más conveniente para el

Así que se prepararon cien carros para que el Príncipe de Chang'an partiera como rehén hacia el Estado de Qi y, a su vez, Qi envió su ejército.

${ }^{17}$ El ejemplo expreso es el de los hijos de los reyes que recibieron el título y potestades de hóu, traducido generalmente como "marqués". 
La anécdota cuya traducción al castellano presentamos nos proporciona un ejemplo privilegiado de la exercitatio persuasiva del período. ${ }^{18} \mathrm{En}$ su artículo titulado "Rhetoric as the Art of Listening", el sinólogo Joachim Gentz destaca el texto precisamente por su valor representativo: "proporciona una brillante ilustración de los principios teóricos del Guiguzi", escribe. ${ }^{19}$ El Guiguæi debe su nombre al homónimo Maestro del Valle Fantasma o de los Fantasmas, el misterioso personaje a quien se atribuye y que vivió a mediados del siglo IV a. C. Considerado durante mucho tiempo como un libro maldito y peligroso, excluido del canon clásico y estigmatizado por pertenecer a una corriente de pensamiento anti-confuciana, acusada de amoral, se mantuvo lejos del interés general durante siglos, relegado en uno de los lados "oscuros" de la cultura china. Reivindicado en las últimas décadas, ha sido presentado como el primer tratado retórico de China y es en sí mismo un enigmático manual teórico que no incluye ejemplos prácticos. La anécdota vendría así a encarnar esos principios si acaso demasiado abstractos.

Gentz cita las observaciones al respecto de Robert Eno, profesor de la Universidad de Indiana y traductor del pasaje. Según Eno, el texto pone de manifiesto una lección fundamental de la teoría retórica china: que la estrategia del suasor debe adecuarse al humor y carácter del gobernante a quien se dirige. "La persuasión no era simplemente cosa de memorizar una retahíla de trucos -concluye Eno-; era un arte, como pretende ilustrar esta anécdota". ${ }^{20}$ Veamos cómo se plasma aquí este arte.

En primer lugar, el episodio presentaría el carácter fundamental de la relación retórica en la China de los Reinos Combatientes: no estamos ante un monólogo, sino ante una interacción dialogada entre el poder y su consejero. No hay virtuosismo elocutivo por parte de un orador, ni especiales florituras literarias, "tan sólo una serie de temas aparentemente sin relación que se emplean para inducir a la reina madre a compromisos de sentido común con ciertos valores y, a partir de estos compromisos, varias preguntas muy sutiles." 21

Arrastrado sin duda por el debate que iniciara en su día François Jullien sobre el Guiguzi y la anti-retórica china, Gentz habla de la aplicación de los "principios manipulativos" por parte del consejero. ${ }^{22}$ Nosotros preferimos hablar de estrategia persuasiva, y conviene advertir hasta qué punto se ajusta al esquema canónico de las partes del discurso. Tras la presentación que hace el narrador de las circunstancias generales, Chu Long arranca su intervención con una particular captatio benevolentiae declarándose viejo y enfermo, presentándose así como una persona inofensiva y que,

\footnotetext{
18 Pujante y Wang (2001: 127-130).

19 Gentz (2014: 1011). La traducción es nuestra.

${ }^{20}$ Gentz (2014: 1013). La traducción es nuestra.

${ }^{21}$ Gentz (2014: 1012). La traducción es nuestra.

22 Gentz (2014: 1012).
} 


\section{TALIA DIXIT 15 (2020), 101-121}

además, no ha venido a hablar del asunto que tiene a la reina Wei tan enfadada. Tan sólo pretende interesarse por su salud, afirma. Esa introducción bajo la forma de una conversación banal trae como consecuencia un efecto psicológico convenientemente subrayado por el narrador: disipa la irritación de la reina y la lleva a un estado de distensión y disposición a escuchar a Chu Long. Después de un par de respuestas secas y algo desabridas, esa distensión permitirá a la reina incluso bromear con su ministro.

El ministro introduce entonces otro tema tangencial, como si desvelase el verdadero motivo de su visita: pide una colocación para su hijo pequeño en palacio. Por razones obvias no podemos esperar narratio ni divisio de ningún tipo, pero, sin que la reina se dé cuenta, una correlación argumentativa ya se ha colado por la puerta trasera: la conversación terminará por vincular el interés del viejo por su hijo menor con la actitud de la reina hacia el suyo. La regente ni siquiera parece muy consciente de que está hablando del Príncipe de Chang'an, el tema por el que no hace tanto amenazaba a sus ministros con escupirles a la cara. El "General de la Izquierda" nos da una lección magistral, justamente, de mano izquierda. Dirigida con aire de ingenuidad, la conversación parece centrarse en una cuestión de afectos.

Desde el punto de vista de la argumentación, Chu Long procede mediante un mecanismo que podríamos describir como disociación y re-asociación de los papeles de su interlocutora como reina y madre o, si se prefiere, de sus responsabilidades públicas y privadas. En primer lugar, va apoyándose en las declaraciones de la mujer para reintroducir el asunto relativo a Chang'an desde un ángulo que parece ignorar las cuestiones de Estado (evitando así herir la susceptibilidad de Wei) y centrarse en la actitud personal de la madre, hasta llevarla a admitir que la sobreprotección que dedica a su hijo menor no le va a ser a éste de ninguna utilidad. En ese punto los intereses de la reina y los de la madre se hacen confluir y el lazo argumentativo se cierra. El brillante ejercicio persuasivo de Chu Long culmina entonces: la enérgica tài bòu se da por vencida, saca las conclusiones por su cuenta y autoriza a proceder con las exigencias de Qi para enviar el ejército en auxilio de Zhao.

El narrador del ZGC concluye su relato de la anécdota con una coda:

Cuando $\mathrm{Zi} \mathrm{Yi23}$ lo supo, dijo:

- Si ni siquiera los hijos de la realeza o los miembros de la familia real pueden mantener su posición de privilegio sin hazañas personales ni obtener un estipendio o conservar su patrimonio sin sacrificio, jcuánto menos podríamos los consejeros! ${ }^{24}$

$23 \mathrm{Zi} \mathrm{Yi} \mathrm{era} \mathrm{una} \mathrm{personalidad} \mathrm{de} \mathrm{Zhao} \mathrm{especialmente} \mathrm{identificada} \mathrm{con} \mathrm{la} \mathrm{virtud.}$

24 Chén. 


\section{TALIA DIXIT 15 (2020), 101-121}

Esta coda devuelve la anécdota al lector implícito, el potencial consejero que aprende de estos ejemplos, y remite a una lectura "superior" del episodio efectuada por otro consejero célebre y en activo, quien saca consecuencias para su propio gremio.

La técnica yīn: teoría y práctica retórica en Lüshi chunqiu

Podemos rastrear la exercitatio retórica durante los Reinos Combatientes en otros documentos de la misma época recogidos en la obra conocida en chino como Lüshi chunqiu [吕氏春秋 lü shì chīn qiü; dicción propuesta: lü shi chun chiu; en adelante LSCQ]. El nombre con que se ha trasmitido la engarzaba en una tradición cuyo origen se hacía remontar al propio Confucio, los chunqiu, una palabra que da nombre al período de la historia china en que vivió el gran filósofo chino, Primavera y otoño, y que serviría a partir de aquel trabajo que se le atribuía tradicionalmente para identificar una serie de escritos cuyos títulos se traducen convencionalmente como "anales" o "crónicas". El título chino de la obra habría, pues, de traducirse bien como "Primavera y otoño del Señor Lü" o bien como "Crónicas" o "Anales del Señor Lü”.

El "Señor Lü" no es otro que Lü Buwei, ${ }^{25}$ un comerciante originario del pequeño Estado de Wei que maniobró hábilmente hasta convertirse en el poder en la sombra en Qin (léase Chin) justo en el momento en que este reino iniciaba su camino hacia la hegemonía. En el año 250 a. C. consiguió instalar en el trono de Qin al rey Zhuangxian. Como resultado de su maniobra, Lü Buwei se convirtió en la mano derecha del rey y detentaría el poder de facto cuando Zhuangxian falleció, tres años más tarde. Su influencia en el reino se mantendría hasta 235, año en que se suicidó, sucumbiendo al panorama de intrigas que se desencadenaron cuando el legítimo heredero del monarca alcanzó la mayoría de edad y asumió resueltamente el poder del reino. Con el tiempo, el entonces príncipe Zheng unificaría China y llegaría a ser conocido para la historia como el Primer Emperador (Qin Shihuang).

En un siglo en que los distintos centros de poder propiciaron un activo mecenazgo de la cultura, y mientras mantuvo el control de la situación, Lü Buwei aspiró a que la corte de Qin adquiriera un prestigio cultural a la altura de su poder militar y político. Para ello reunió a un nutrido grupo de shi 士, ${ }^{26}$ a quienes con buenos argumentos podríamos describir como hidalgos ilustrados: vástagos de una nobleza de origen guerrero, desclasada y empobrecida, trataban de ganarse la vida mediante el estudio, viajando de corte en corte y ofreciéndose como consejeros

25 Una revisión biográfica detenida de este interesante personaje puede encontrarse en Knoblock y Riegel (2000: 1-12).

${ }^{26}$ Knoblock y Riegel (2000: 13). 
políticos. Conocidos como biànshi (biàn 辩 significa argumentación) cuando se quiere enfatizar sus capacidades argumentativas, es la clase social a la que perteneció el propio Confucio. A estos intelectuales-caballeros itinerantes les propuso poner su conocimiento por escrito hasta completar un tratado enciclopédico sobre el arte del gobierno. Por muchas razones, la teoría fundamental del libro sostiene un alegato para que el gobernante respete a los shì y deposite en ellos su confianza y la administración del Estado. Pero su contenido iba mucho más allá. Según el historiador Sima Qian, el plan concebido por Lü habría de "abarcar la totalidad de los asuntos del Cielo y de la Tierra, de las diez mil cosas, y del pasado y el presente" ${ }^{27}$. El resultado tal como lo conservamos ronda los ciento veinte mil caracteres y, según Sima Qian, se expuso en el mercado de Xianyang, la capital de Qin, con un orgulloso mensaje destinado a los sabios itinerantes: si alguno de ellos era capaz de añadir o quitar un simple carácter recibiría mil medidas de oro. Sin embargo, a pesar de la reivindicación de Sima Qian, los estudios prueban que, si bien conservamos la obra completa prácticamente tal como Lü la dejó a su muerte, el proyecto está inacabado con arreglo a su concepción original. ${ }^{28}$

A menudo se ha caracterizado al LSCQ como una "miscelánea", es decir, un texto "ecléctico" o "sincrético", atribuido por más señas a una "escuela mixta" (zájia ). Pero sería un error entender la obra como un simple refrito o centón de extractos procedentes de otras obras y, por tanto, carente de originalidad de pensamiento. Lü Buwei pretendió crear en torno a la obra una escuela filosófica independiente con la misión de diseñar una teoría para el imperio unificador ${ }^{29}$ cuyo embrión veía en Qin - y que acabaría realizando el príncipe Zheng, de quien Sima Qian insinúa que era, en realidad, hijo del propio Lü. Destinado genéricamente a aquellas personas en posiciones de autoridad o que aspirasen a ocuparlas, ${ }^{30}$ algunos estudiosos alegan que su destinatario último era precisamente Zheng, considerando la obra así un verdadero "espejo de príncipes". ${ }^{31}$

Ese ambicioso proyecto se sostiene sobre un complejo plan cosmológico regido por un "pensamiento correlativo", en el que los actos de los hombres se articulan y acompasan con el curso de los Cielos y la secuencia de las estaciones en la Tierra, replicando sus grandes patrones y comportándose así como microcosmos. ${ }^{32} \mathrm{~A}$ esa correlación responde la tripartición de la obra, dividida en 12 libros de jì (registros o "almanaques" que organizan los temas de acuerdo con la estación del año y proporcionan la estructura teórica y general de la obra), 8 de lăn (“exámenes" o

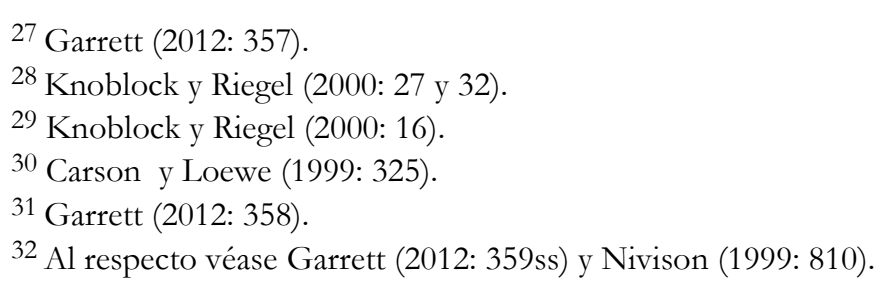


“inspecciones") y 6 de lìn (palabra para cuya traducción quisiera recuperar en este contexto el latinismo de "disputaciones"), que se corresponden respectivamente con la tríada compuesta por Cielo, Tierra y Ser humano. ${ }^{33}$

En un artículo en la revista Rhetorica, la profesora estadounidense Mary M. Garrett reivindicó la relevancia retórica del libro, generalmente menospreciado desde este punto de vista, subrayando precisamente su particular combinación de teoría y práctica, sistema y ejemplo:

In these discussions it presents and evaluates examples of successful and unsuccessful attempts at persuasion, of sophistry, and of unethical rhetoric, and it also lays out a system of rhetorical precepts that are motivated, in large part, by its ontology. In addition, because the book is itself a persuasive document addressed to a specific person, its own rhetorical practices can be held up to the theory it proclaims. ${ }^{34}$

Un aspecto de particular importancia que Garrett subraya en el planteamiento retórico de LSCQ es su análisis del auditorio, teniendo en cuenta que en su contexto histórico el deliberans (es decir, el consultante o auditorio del ejercicio de persuasión política) es, por excelencia, el soberano que escucha a su consejero:

The question (...) is why persuasions that are unethical or wrong are, nonetheless, accepted by the listener (...) For the LSCQ there are two answers; the deficiencies of the audience or the inadequacies of the persuader. ${ }^{35}$

El LSCQ analiza esas "deficiencias del auditorio" que, a nuestro juicio, poseen un interés que trasciende el contexto histórico estricto. ${ }^{36}$ Júzguese. En primer lugar, puede que el discurso llegue a oídos de alguien estúpido sin remedio: eso condena a la inutilidad el trabajo del más hábil de los consejeros. Habitualmente, sin embargo, el consultante está afectado por otro tipo de defectos: con frecuencia no puede deshacerse de sus prejuicios, deseos u obsesiones y eso limita seriamente su capacidad crítica. En ciertos casos, malinterpreta los consejos porque carece de preparación y capacidad analítica. En último extremo, el auditorio sencillamente no quiere escuchar la verdad. En el marco de las relaciones de poder de la antigua China el resultado no solamente supone el fracaso del discurso persuasivo, sino que, como dijimos, puede acarrear un riesgo extremo para el consejero. El margen de éxito se mueve, diríamos, entre la segunda y la tercera de las posibilidades mencionadas. De

33 Información detallada puede encontrarse en Knoblock y Riegel (2000: 32ss) y Carson y Loewe (1999: 324-5).

34 Garrett (2012: 355). Los subrayados son nuestros.

35 Garrett (2012: 368).

36 Garrett (2012: 368-9). 
ahí la importancia capital del dominio de las técnicas retóricas por parte del consejero y de su capacidad para hacerlas aceptables a un interlocutor poco capaz, obstinado u hostil.

Una de esas técnicas es la que se describe en la sección correspondiente a las “inspecciones” del LSCQ, en el libro 15, capítulo 5, titulado Shùn shuō 顺 说. Nuestra traducción se basa en el texto fijado por la edición bilingüe de Knoblock y Riegel, páginas 356 y 357, quienes, a su vez, traducen el título del capítulo al inglés como On making persuasions agreeable.

La teoría propiamente dicha se recoge en el pasaje 5.1 del capítulo, en nuestra traducción:

El buen persuasor es como un guerrero hábil:

se apoya en la fuerza de los otros para hacerse fuerte, se apoya en que vienen para ayudarles a venir, se apoya en que van para ayudarles a ir.

No exhibe su proceder ni su apariencia:

engendra y desarrolla conforme lo hacen ellos;

se apoya en las palabras de los otros

y sus propias palabras son como sombras o ecos.

Prospera y declina conforme lo hacen ellos;

con estos medios alcanza su propósito.

Por muy fuertes y capaces que sean,

controla su voluntad.

Cuando alguien grita a favor del viento, no es que tenga más voz; cuando alguien sube a un altozano para otear, no es que sus ojos vean mejor: la ventaja se la da aquello en lo que se apoya.

Traducimos la primera parte del pasaje como líneas versales porque esta parte está rimada en el original. Esa naturaleza poética (y mnemotécnica) que adopta la forma incluye también su razonable dosis de oscuridad. Además de tratar de hacer justicia a la belleza de la expresión, que invitamos a apreciar, la licencia poética nos permite a nosotros emplear el término "persuasor", que la Real Academia admite sólo como adjetivo, con valor sustantivo.

Cabe observar, en todo caso, que la lección teórica que se imparte se articula sobre un símil en el que los shì son protagonistas: allí donde traducimos "guerrero" se encuentra el carácter correspondiente a este colectivo. El shì se desdobla como un Jano bifronte: para traducirlo al castellano hemos tenido que optar por una de sus dos caras. Su dominio de las artes marciales se convierte en 
ilustración de su perfil consejístico y, por esta vía, el discurso persuasivo del consejero se asimila al combate del caballero tan estrechamente, que no queda claro en qué momento los predicados pasan a depender de uno a otro sujeto. En su doble papel de combatiente y de consejero se recomienda al shì una técnica concreta (yìn 因) que consiste en "apoyarse" o "basarse en" los movimientos de quien tiene enfrente. Hemos destacado en cursiva las veces en que el carácter de yìn aparece expresamente en el texto. En la parte versificada es notorio su uso anafórico. Cuando aparece, una sola vez, en la coda en prosa lo hace para cerrar el texto, redondeándolo, y aclarar el sentido de su uso en la parte rimada.

Nuestra traducción del pasaje 5.1 es parcial. En la edición de Knoblock y Riegel, el texto cuya traducción ofrecemos viene precedido de unas líneas en prosa cuya pertenencia al Shùn shuō o al final del capítulo anterior está a debate. Knoblock y Riegel aceptan la lectura de Chen Qiyou, para quien deben abrir el capítulo 5. En ellas se atajan las posibles excusas del consejero ante un fracaso aduciendo deficiencias del auditorio ("Cuando no se hace caso a los consejos, la responsabilidad no recae sólo en el consultante: también recae en el asesor”) y se hace hincapié en la idea de que el consejero hábil no tiene necesidad de emplear fuerza ninguna, no debe violentar a su oyente: "¿Qué falta hace recurrir a la fuerza? Quien tiene que recurrir a la fuerza para convencer, se rebaja”, se dice allí. Y es cierto que esa idea se engarza con naturalidad con el símil del guerrero: igual que el luchador avezado se sirve del impulso agresivo del contrario o de su resistencia para derrotarle, sin malgastar su fuerza propia, el buen suasor no debe tratar de contrariar o forzar la opinión de su auditorio; en realidad, no tiene más que amoldarse a él, seguir la estela discursiva de su interlocutor ("no exhibe su proceder ni su apariencia"). El consejero debe renunciar a todo protagonismo o lucimiento personal: sus propias palabras no deben ser más que "sombras o ecos" de las de ese interlocutor. La eficacia se presenta así como aprovechamiento astuto de la inercia ajena.

A diferencia del Guiguri, un manual teórico sin ejemplos prácticos, en LSCQ se combinan adecuadamente teoría y práctica, ars y exercitatio, ilustrando con anécdotas los planteamientos doctrinales. De este modo, el símil del shì combatiente y su técnica yin están desarrollados a renglón seguido por varios episodios narrativos. De ellos traducimos el primero y más extenso, el pasaje 5.2. En él vuelve a repetirse el planteamiento indirecto, de aparente respeto a las exigencias del auditorio y también, como una moraleja, la idea de derrotar por ese medio a alguien más fuerte que uno mismo - algo que puede entenderse perfectamente en dependencia de la relación de poder entre el consejero-David y el rey-Goliat. La escena se desarrolla esta vez en el Estado de Song, otro de los siete reinos combatientes, bajo el rey Kang, quien ocupó el trono entre el año 328 y el 286: 
Hui Ang tenía audiencia con el rey Kang de Song. El rey Kang dio un enérgico pisotón en el suelo, carraspeó y le espetó:

- Lo que a mí me gusta son hombres con coraje y fuerza, no humanistas y moralistas. ¿Sobre qué pretende aleccionarme nuestro huésped?

Hui Ang respondió:

- Supongamos que poseo el método para que una persona, por coraje que tenga, no consiga atravesarte cuando intenta apuñalarte ni, por fuerte que sea, acierte cuando intenta atacarte. ¿No estaría Vuestra Majestad interesado en conocerlo?

—Estupendo! —dijo el rey—. Eso es lo que quiero escuchar.

Dijo Hui Ang:

- Pero, aunque el agresor no consiga atravesarte cuando te apuñala ni acertar cuando te ataca, puede todavía de humillarte. Poseo el método para que, por coraje que tenga, ni siquiera se atreva a apuñalarte ni, por fuerte que sea, se atreva siquiera a atacarte. ¿No estaría Vuestra Majestad interesado en conocerlo?

—Estupendo! — dijo el rey—. Sobre eso sí que quiero saber.

Dijo Hui Ang:

- Pero, aunque esa persona no se atreva a apuñalarte ni se atreva a atacarte, eso no significa que no tenga la intención. Poseo el método para conseguir que la gente no albergue en absoluto esa intención. ¿No está interesado Vuestra Majestad en eso?

—Estupendo! — dijo el rey—. No espero otra cosa.

Dijo Hui Ang:

- Pero, aunque la gente no tenga esa intención, puede que en su corazón no sienta aún amor hacia los demás y voluntad de hacer el bien. Poseo el método para lograr que todos los hombres y mujeres del mundo sin excepción deseen fervientemente sentir amor y hacer el bien. Eso es, sin duda, mejor que tener coraje y fuerza, superior en cuarto grado. ¿No estaría Vuestra Majestad interesado en eso?

Respondió el rey:

—Es lo que quiero conseguir.

Hui Ang repuso:

-Pues eso es lo que enseñan Confucio y Mozi. Confucio y Mozi no eran soberanos de territorios ni tenían funcionarios a su servicio, y sin embargo todos los hombres y mujeres del mundo estiraban el cuello y se empinaban esperando sus atenciones y beneficios. Hoy día, Vuestra Majestad es el dueño de diez mil carros: si tuviera esa misma aspiración, entonces todos dentro de las cuatro fronteras disfrutarían de sus beneficios y Vuestra Majestad sería mucho más grande que Confucio y Mozi.

El rey de Song no supo qué contestar y Hui Ang se apresuró a salir.

—QQué argumentación! ¡Cómo me ha obligado a admitirla nuestro invitado! — confesó el rey a sus cortesanos-.

El rey de Song era un soberano mediocre, pero su corazón podía aún ser convencido con la técnica yin del "apoyo". Con ese arte, el pobre y humilde 
puede superar al rico y noble, el pequeño y débil puede controlar al fuerte y poderoso.

Al comienzo de la anécdota, el rey Kang da a Hui Ang el tratamiento de kè 客: invitado, huésped o forastero. En otras palabras, reconoce en él a uno de los sabios itinerantes que venían tratando de impresionarle para que les contratase. Hui Ang es un shi. El encuentro debió de tener éxito para Hui Ang, porque se le conoce precisamente como consejero de este rey - cuya resistencia venció sin enfrentarse directamente a ella en ningún momento.

El rey está caracterizado aquí como un tipo enérgico de modales bruscos y violentos, muy poco dispuesto a escuchar los sutiles razonamientos que presupone en Hui Ang, precedido por la fama de los consejeros itinerantes, educados en las tradiciones escolares fundadas por los grandes maestros del período Primavera y otoño, Confucio y Mozi. Eso que presume el rey con lo que viene a sermonearle Hui Ang se denomina en el original rén yì 仁义, buena voluntad y justicia, valores característicos de estas escuelas y que traducimos como "humanistas y moralistas" para actualizar el alcance de su significado - desde el punto de vista del rey. Desde sus alturas, la actitud del rey de Song es agresiva, desafiante, exhibe desprecio por los shi. Esa posición de partida del todopoderoso soberano añade valor al logro final de Hui Ang, quien le ha obligado a aceptar que de las doctrinas "buenistas" de los grandes filósofos puede obtenerse lo que no consigue el recurso a la fuerza: no ya prevenir y derrotar los ataques, sino sencillamente hacer imposible que se produzcan.

Ante todo, Hui Ang respeta las expectativas e intereses del soberano ("Eso es lo que quiero escuchar", corrobora él). El ingrediente de simulación o distracción que vimos en la estrategia de Chu Long vuelve a estar aquí presente, pero, en lugar del mecanismo de disociación y re-asociación de las identidades de la tài hòu que plantea Chu Long, la cancamusa, la maniobra de distracción, el "nudo" que permitirá apretar el lazo argumentativo y atrapar en él a su interlocutor radica esta vez en la ambigüedad de una palabra, dào 道, que se ha transcrito tradicionalmente como tao y traducimos aquí como "método" — pero que se define como lo indefinible en el Daodejing, texto fundacional del taoísmo. Hui Ang explota la célebre imprecisión del vocablo o, si se quiere, su capacidad de evocación. Puesto que se habla de combatir la violencia, esa palabra puede sugerir en la mente de Kang un sistema defensivo: una estrategia, una táctica, un arma o incluso un conjuro mágico. Esa ambigüedad calculada permite que Hui Ang mantenga al rey dentro de lo que "quiere escuchar" mientras lo prepara para aceptar lo que no quería oír desde el principio.

Así pues, hablando de fuerza y de coraje, no de moral y humanismo, Hui Ang consigue que el rey admita que, si las doctrinas de Confucio y Mozi se instalasen en los corazones de la población (objetivo último del shì y precisamente la prédica 


\section{TALIA DIXIT 15 (2020), 101-121}

contra la que estaba prevenido el monarca), eso significaría una victoria "superior en cuarto grado" contra los enemigos del rey. Ya no se trata de hacer que fracasen los ataques potenciales que se perpetren contra él (victoria en primer grado), o de que sus enemigos no se atrevan a atacarle (segundo grado), o -mejor todavía- de que ni siquiera tengan interés en hacerlo (tercer grado): mucho más seguro y eficaz que todo eso es que incluso sientan afecto y amor hacia él, voluntad de protegerle. El "método" defensivo más eficaz y poderoso, más que los arcos y las flechas, es la educación moral y la cultura de la paz. Ésa es la garantía más firme de la derrota del enemigo.

Esta misma anécdota, con algunas variantes, aparece en otras dos obras antiguas: en el Huainanzi, capítulo 12 (Daoying), pasaje 9, y en el Liezi, capítulo 2 (Huang Di), pasaje 21. Este último pasaje ha sido traducido al castellano por Iñaki Preciado. ${ }^{37}$ Significativamente, ninguna de las dos versiones alternativas recoge las repetitivas y entusiastas respuestas del rey (“¡Estupendo!”) a cada proposición de Hui Ang. Es propia del narrador del LSCQ una presentación interactiva del episodio que sobredimensiona el diálogo donde las otras versiones incluyen una sola interrupción del rey partiendo en dos un monólogo de Hui Ang relativamente extenso. Este narrador, en cambio, ha optado por una construcción reiterativa y pendular, donde la conversación avanza a base de preguntas y respuestas: a cada intervención, pequeñas modificaciones de gran valor significativo por parte de Hui Ang obtienen una respuesta prácticamente igual por parte del rey Kang. De este modo, el narrador del LSCQ parece dibujar una esgrima. En su versión, Hui Ang consigue la victoria persuasiva final, nunca mejor dicho, gradualmente, paso a paso: cada uno de los cuatro pasos argumentativos que admite Kang es una victoria parcial del shi, un asalto ganado. Y, al revés, cada “¡Estupendo!” del rey Kang constituye, irónicamente, una derrota por puntos.

No menos ilustrativo resulta el hecho de que en ninguna de las dos versiones alternativas aparece la coda con la que se remata aquí la anécdota ${ }^{38}$ ni se establece conexión alguna con la técnica del "apoyo". El responsable de la versión del LSCQ quiso, evidentemente, subrayar el interés didáctico del episodio: ilustrar la técnica yìn y su fuerza. El ejemplo es una lección para estudiantes de consejística que redondea la teoría expuesta por medio del símil del guerrero: comprobamos en la práctica la capacidad de un consejero para someter al dragón que se esconde en el rey al que sirve y aconseja.

37 En el Huainanzi, Hui Ang aparece identificado como Hui Meng. En la traducción de Preciado aparece como "Hui Yang”, creemos que erróneamente. Cf. Preciado (20197: 75-76).

${ }^{38}$ La anécdota concluye en el Liezi i con el comentario admirado del rey Kang. En el Huainanzi se añade una apostilla atribuida a Laozi sobre un tema muy diferente: el valor y el miedo. 


\section{TALIA DIXIT 15 (2020), 101-121}

\section{A modo de conclusiones}

Si bien se mira, hay un aire de familia entre las dos anécdotas que hemos comentado. Ambos suasores, Chu Long y Hui Ang, deben enfrentarse a un auditorio poderoso y, para colmo, desconfiado e incluso hostil. Se ven así obligados a desplegar una estrategia indirecta que, desdeñando cualquier virtuosismo elocutivo, renunciando de antemano a la elocuencia, pretende en todo momento no violentar ni contrariar las expectativas de su singular auditorio. A ambos se les concede el derecho a la palabra, pero ni siquiera pueden decidir el tema sobre el que hablar. De hecho, deben evitar incluso alarmar a su interlocutor o hacerle sospechar que se está tratando de un tema hacia el que, de entrada, el poderoso ha mostrado disgusto. Ante esa actitud recelosa y suspicaz, la aproximación al tema se hace desde otro lado, en apariencia ajeno y distante al que finalmente emergerá como asunto central del interés por parte del consejero. Ese otro extremo temático se va haciendo coincidir con el que interesa al consejero de forma gradual, sin que el interlocutor sea siquiera consciente de que eso está sucediendo. Cuando se hacen coincidir, se aprieta el lazo.

De hecho, ambos episodios podrían ser intercambiables como ejemplos tanto del Guiguzi como de la técnica yìn, y esto es así porque comparten ciertas características que consideramos emblemáticas del discurso persuasivo tal como se teoriza en la Antigüedad china y, más en concreto, durante los Reinos Combatientes, resultado del particular contexto de la exercitatio retórica y la desequilibrada relación de poder que se establece entre soberano y consejero. Si aceptamos, como sugiere Gentz, que el pasaje del ZGC sirve de ilustración a los esquemas teóricos del Guiguæi, esa intercambiabilidad que argüimos podría reforzarse más aún si se considera que ese "apoyarse" o "basarse en" que se ilustra en LSCQ, planteado con un símil bélico o militar, recuerda a la actitud de reserva y espera que se recomienda como estrategia en el Arte de la guerra de Sunzi, con quien se ha relacionado estrechamente al Guiguri y al autor a quien se atribuye. ${ }^{39}$ Compárese el símil del guerrero con este pasaje de Sunzi en la traducción castellana de Albert Galvany:

El buen estratega somete las fuerzas enemigas sin combatirlas, toma las fortificaciones enemigas sin asaltarlas, desmembra los Estados rivales sin permitir que las campañas militares se prolonguen. De este modo, puede conquistar el mundo entero conservando sus fuerzas; sus regimientos no desfallecen y sus riquezas se mantienen intactas. ${ }^{40}$

${ }^{39}$ Véase Conde y Zhao (2018: 28).

${ }^{40}$ Galvany (2017: 121). 


\section{TALIA DIXIT 15 (2020), 101-121}

Al menos desde el punto de vista retórico, si no conociéramos anécdotas ejemplificadoras como las que se nos cuentan en el LSCQ o en el ZGC, esas estrategias parecerían provocadoras y arriesgadas ocurrencias de paradojismo, sin sostén en la realidad.

Finalmente, aunque la historia de Chu Long no compromete ningún tipo de valor moral y resulta evidente la influencia confuciana y moísta en el pasaje de Hui Ang, eso no invalida el principio general de que la teoría del discurso persuasivo dejaba en suspenso los principios éticos. La ejecución de la técnica yīn puede implicar circunstancialmente recurso a comportamientos que entran en conflicto con los principios morales del orador. El LSCQ detalla cómo Mozi, cuya austeridad desdeñaba los vestidos de moda y los deleites musicales, se vistió con atuendos llamativos y tocó la flauta para conseguir una audiencia con el rey de Chu, o menciona que Confucio se valió de Mi Zixia, un prototipo de la depravación sexual, para conseguir ser presentado a un personaje, la señora $\mathrm{Li}$, que era su verdadero objetivo $^{41}$. Incluso el LSCQ se rinde en la práctica a la evidencia de que es el recurso adecuado el que consigue el efecto deseado, absteniéndose de presentar reproches de orden moral.

JUAN LUIS CONDE

Universidad Complutense de Madrid

DAVID SEVILLANO LÓPEZ

Universidad Complutense de Madrid

ZIQIAO MENG

Universidad Carlos III de Madrid

${ }^{41}$ Garret (2012: 371). 


\section{BIBLIOGRAFÍA}

Carson, M. y Loewe, M. (1993), “Lü shih ch'un ch'iu”, en Loewe, M. (ed.), Early Chinese Texts. A Bibliographical Guide, Berkeley: University of California, pp. 324330

Conde, J.L. y Zhao, L. (2016), "Suasoria bajo presión: traducción y comentario al capítulo 3 del Han Feizi (Aportación al estudio de la retórica política en China clásica, 2)", Rétor 6 (1): 28-50.

Conde, J. L. y Zhao, L. (2018), "Manipular y persuadir en la antigua China: el Guiguzi. Traducción del capítulo 9", Talia Dixit 13: 27-43.

Fan, X. (2018), Zhan Guo Ce Jian Zheng, vol. 3, Shanghái: Shanghai Classics Publishing House.

Galvany, A. (trad., intr. y notas) (2017), Sunzi. El arte de la guerra, Madrid: Trotta.

Garrett, M. M. (2012), “What Need Is There of Words?' The Rhetoric of Lü's Annals (Lüshi chunqiu)", Rhetorica 30 (4): 354-374.

Gentz, J. (2014), "Rhetoric as the Art of Listening: Concepts of Persuasion in the First Eleven Chapters of the Guigur?", Asia 68 (4): 1001-1019.

Jullien, F. (1999), Tratado de la eficacia, Madrid: Siruela.

Knoblock, J. y Riegel, J. (ed., trad. y estudio) (2000), The Annals of Lü Buwei, Stanford: Stanford University Press.

Li, Ch. (2017), "La retórica china: historia y cultura”, Rétor, 7 (1): 21-39.

Nivison, D. Sh. (1999), “The Lü Shi Chun Qiu and Correlative Thinking”, en Loewe, M. y Shaughnessy, E. L., Cambridge History of Ancient China, Cambridge: Cambridge University Press, pp. 808-812.

Preciado, I. (trad., intr. y notas) $\left(2019^{7}\right)$, Lie Zi. El libro de la perfecta vacuidad, Barcelona: Kairós.

Pujante, D.-Wang, M. (2001) “¿Hubo una práctica retórica en la antigua China? Algunos ejemplos del arte de persuadir en su tradición literaria", Estudios de literatura 26, 121-136.

Tsien, Ts.-hs. (1993), "Chan kou ts'e”, en Loewe, M. (ed.) Early Chinese Texts. A Bibliographical Guide, Berkeley: University of California, pp. 1-11.

Wu, H. y Swearinguen, C. J. (intr., trad., notas, coment.) (2016), Guiguri: China's First Treatise on Rhetoric. A Critical Translation and Commentary, Carbondale: Southern Illinois University Press. 